\title{
Interactive comment on "32-year record-high surface melt in 2019/2020 on north George VI Ice Shelf, Antarctic Peninsula" by Alison F. Banwell et al.
}

Alison F. Banwell et al.

alison.banwell@colorado.edu

Received and published: 8 January 2021

Please see attached Supplementary file.

Please also note the supplement to this comment:

https://tc.copernicus.org/preprints/tc-2020-309/tc-2020-309-AC2-supplement.pdf

Interactive comment on The Cryosphere Discuss., https://doi.org/10.5194/tc-2020-309, 2020. 